\title{
A case of spondylolysis and spondylodiscitis in a child
}

\begin{abstract}
Spondylolysis is a common reason of low back pain in children and adolescents, which can be caused by some predisposing factors and also is associated with some environmental factors. On the other hand spondylodiscitis is an uncommon diagnosis in children caused by different agents and according to the configuration of the vertebrae and adjacent structures in children the mechanism of pathogenesis is different from adults. We present a case of spondylolysis and spondylodiscitis in a 3year-old girl with low back pain.
\end{abstract}

Keywords: spondylolysis, spondylodiscitis, magnetic resonance imaging
Volume 3 Issue 5 - 2017

\author{
Angela Guarnizo, Sonia Bermudez, Nicolas \\ Useche \\ Department of Radiology, Hospital Universitario Fundación \\ Santa Fe de Bogotá, Colombia
}

Correspondence: Angela Guarnizo, Department of Radiology, Hospital Universitario Fundación Santa Fe de Bogotá, Colombia, Email apguarnizo@gmail.com

\section{Introduction}

Spondylolysis refers to the unilateral or bilateral fracture of the pars interarticularis, it is associated with predisposing factors which together with straight posture, gait and repeated load of the lumbosacral spine predispose to develop the disease. ${ }^{1}$ Most cases are asymptomatic. The prognosis is usually benign with conservative treatment.

Of all infectious bone diseases, the prevalence of spondylodiscitis is $2-4 \%$ in children less than 3years of age ${ }^{2}$ and the incidence of all spinal infections in people aged less than 20years is 0,3 per 100,000 cases peryear. ${ }^{3}$ The symptoms in children usually are refuse to sit or walk, but in most cases are unspecific and delay the diagnosis.

\section{Case presentation}

A 3year-old girl presented with a history of low back pain. The initial $\mathrm{X}$ ray showed spondylolysis and minimal anterior spondylolisthesis at L5-S1 (Figure 1). Lumbar MRI showed extraforaminal disc protrusion with intervertebral disc height loss, vertebral endplate signal abnormality in L4-L5 vertebral bodies, left soft tissue abnormal signal from L2 to S1 and medial aspect of psoas muscle (Figure 2). CT-guided core biopsy of paravertebral soft tissue, confirmed diagnosis of spondylodyscitis.

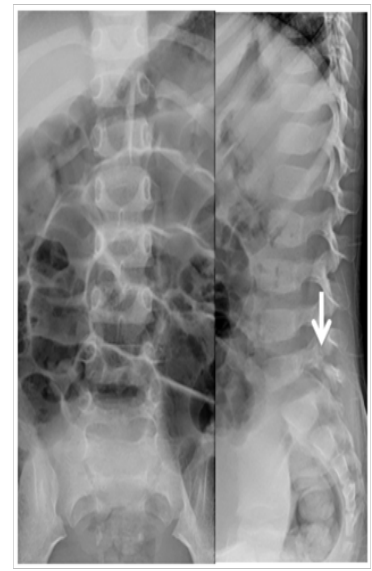

Figure I Lumbar spine AP and lateral x-rays, showing spondylolysis (arrow) and minimal anterior spondylolisthesis at L5-SI.

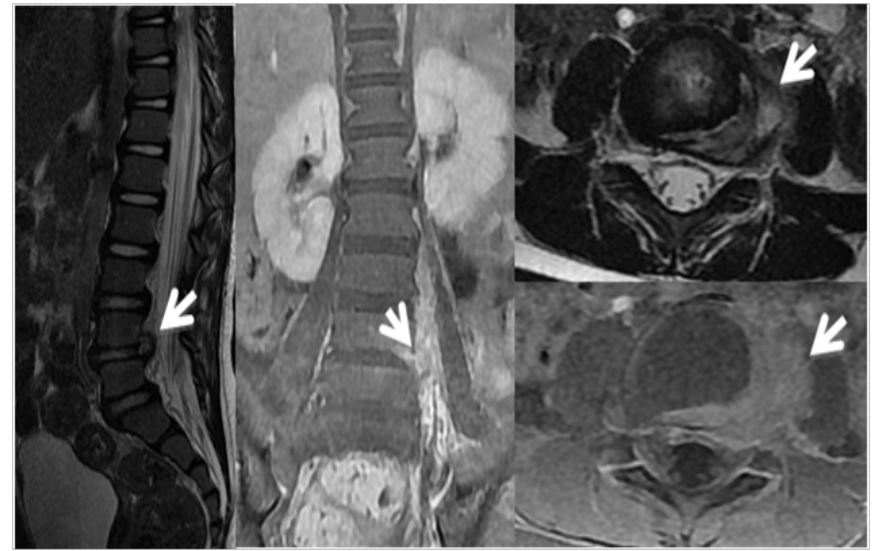

Figure 2 Lumbar MRI Sagittal SSFSE T2, coronal SSFSE TI fat sat with contrast, axial SSFSE T2 and SSFSE TI fat sat with contrast showing left L4-L5 foraminal and extraforaminal disc protrusion with left soft tissue abnormal signal and contrast enhancement from L2 to SI and medial aspect of psoas muscle (arrows).

\section{Discussion}

Spondylolysis pathogenesis: the posterior arc is not completely ossified and the intervertebral disc is very elastic. The pars interarticularis is susceptible to failure due to fatigue caused by tension and shearing forces. The predisposing factors include: hereditary, vertebral dysplasia-spina bifida, elongation of the facets, anomalies of the soft tissues and physis and sacropelvic morphology. ${ }^{1}$ The key diagnostic feature is fracture of the pars interarticularis.

In spondylodiscitis the spine is involved through hematogenous spread or via contaminated contiguous source. The spinal infection begins in the vertebral body near to the endplate in the form of microabscesses, through the vascular channels the infection spreads from the endplate to the disc, vertebral bodies, epidural space and paravertebral soft tissues. ${ }^{3}$

Imaging findings show bone marrow involvement with T1 hipointensity, T2 hiperintensity and enhancement with contrast; most marked in the endplates at the infected level. The disc involvement usually shows disc space narrowing, T2 hyperintensity and enhancement (Figure 3). In some cases irregular endplates and vertebral 
body destruction may be present. Involvement of paravertebral soft tissues may show enhancement and abscess formation. ${ }^{4}$

The differential diagnosis includes degenerative disc changes, acute herniation with adjacent inflammatory soft tissue changes and tumors with propensity to cross the disc space such as chordoma. ${ }^{4}$ According to the age of the patient, the severity of the symptoms and the grade of displacement the treatment for spondylolysis can be conservative with restriction of activities and follow up every 6-12months or surgical in case of persistence of symptoms despite conservative treatment. ${ }^{1}$

Parenteral wide spectrum antibiotics for 3 weeks, followed by oral antibiotics are the treatment of choice in spondylodiscitis. Surgical treatment is recommended for immunocompromised patients or in cases of major vertebral body destruction with consecutive instability, epidural abscess or progressive neurological impairment. ${ }^{3}$

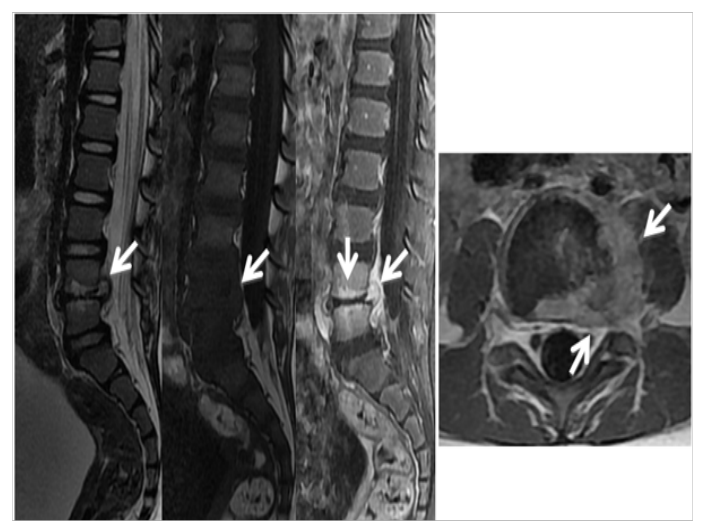

Figure 3 Lumbar MR Sagittal SSFSE T2, SSFSE TI, SSFSE TI fat sat with contrast and axial SSFSE TI fat sat images showing disc protrusion at L4-L5, intervertebral disc height loss and decreased signal, vertebral endplate signal abnormality and L4 and L5 vertebral bodies edema. Post-contrast images show avid enhancement of the endplates and left paraspinal soft tissues (arrows).

\section{Conclusion}

Spondylolysis and spodylodiscitis should be considered in children who refuse to sit or walk or with low back pain. MRI is the modality of choice to make the diagnosis.

\section{Acknowledgements}

None.

\section{Conflict of interest}

Author declares that there is no conflict of interest.

\section{References}

1. Mora-de Sambricio A, Garrido-Stratenwerth E. Spondylolysis and spondylolisthesis in children and adolescents. Revista Española de Cirugía Ortopédica y Traumatología. 2014;58(6):395-406.

2. Waizy H, Heckel M, Seller K, et al. Remodeling of the spine in spondylodiscitis of children at the age of 3years or younger. Arch Orthop Trauma Surg. 2007;127(6):403-407.

3. Tyagi R. Spinal infections in children: A review. Int Orthop. 2016;13(4):254-258.

4. Diehn FE. Imaging of spine infection. Radiol Clin North Am. 2012;50(4):777-798. 